\title{
Knowledge translation in global health
}

\author{
Ariel Pablos-Mendez, ${ }^{1}$ Somsak Chunharas, ${ }^{2}$ Mary Ann Lansang, ${ }^{3}$ Ramesh Shademani, ${ }^{1}$ \& Peter Tugwell ${ }^{4}$
}

\begin{abstract}
The Ministerial Summit on Health Research in November 2004 and the World Health Assembly in May 2005 called for more research in developing countries to strengthen health systems and to help achieve the Millennium Development Goals (MDGs) by 2015
\end{abstract} $(1,2)$. Research, we believe, has to be a component of a strategic process rather than an end in itself. Ill-health and premature deaths from preventable causes persist, especially among poor children and women, in spite of available cost-effective interventions (3), in part because there is a gap between what is known and what gets done in practice, i.e., the 'know - do' gap. This gap between available knowledge and its application in policy and practice is not new, but systematic approaches to address it are urgently needed. New approaches should promote the use of best available scientific evidence and strategic research. They should also harness field experience and innovation with an emphasis on problem-solving to address priority health problems, both at the global and local levels. To bridge the know-do gap, knowledge must be leveraged effectively to achieve better health (4). The generation and sharing of knowledge are necessary steps in its effective application.

Global debate continues on how this gap can be bridged. Evidencebased medicine and health policy represent genuine efforts to ensure that the best evidence informs policy and practice, but much remains to be done. Developing countries are underrepresented in randomized controlled trials and systematic reviews. This under-representation contributes to the mismatch between best evidence and what is relevant, applicable, feasible and affordable in such countries (5). The situation is even more tenuous beyond the clinical setting, given the complex nature of public health and policy, where questions rarely lend themselves to traditional research approaches.

There is debate on what constitutes best available evidence and how to harness it in policy and practice. The research literature refers to a hierarchy of evidence from systematic reviews of randomized controlled trials, to common sense and expert opinion. Moreover, the definition of knowledge is sometimes used interchangeably with that of information or evidence. It has been argued that information is explicit and factual, while knowledge results from the integration of information with belief and context. This implies that while information can flow easily, knowledge is embedded in people (6). The perception of knowledge differs according to culture. Ultimately, it is knowledge that drives people to act.

What makes us believe in an idea enough to transform it into decisions and actions? In the context of strengthening health systems in developing countries, it is timely to revisit basic conceptions of evidence, information and knowledge. Beyond research and the various sources of useful knowledge, the key challenge lies in understanding the steps and processes involved in decisionmaking and implementation. These are complex and influenced by many factors including the user's context, the presence (or lack) of an enabling environment, perceived relevance and type of knowledge in question. Linear, push-pull models imply that knowledge is a commodity that flows along a simple gradient from one group to another. More interactive models account for the complexity of individual reasoning and how groups of people solve problems. In order to bridge the knowdo gap, we need to better understand the means by which knowledge is translated into action. The debate needs to move from the question of what constitutes evidence to problem-solving in policy and practice. The challenge of effective knowledge translation is most apparent in the developing world, but it is present in all countries and their health systems.

A Bulletin theme issue, to be published in the second half of 2006, will focus on the subject of knowledge translation in global health. Commissioned papers will describe the various ways in which knowledge is produced and shared, and depict elements of, and facilitators for, knowledge translation in health. The theme issue will also provide examples of how effective knowledge translation is integral to the research, development, and management of health systems, and how it is affected by funding mechanisms. The Bulletin will also consider your papers on these topics, particularly for the following sections: Lessons from the Field, Policy and Practice, and Perspectives. The deadline for submission is 1 December 2005.

1. World Health Organization. World Report on Knowledge for Better Health. Geneva, 2004.

2. World Health Organization. Health and the Millennium Development Goals. Geneva, 2005. (530.1).

3. Jones G, Stekettee RW, Black RE, Bhutta ZA, Morris SS and the Bellagio Child Survival Study Group. How many child deaths can we prevent this year? Lancet 2003;362:65-71.

4. Canadian Health Services Research Foundation. Leveraging Knowledge: tools \& strategies for action. Report of the 7th CHSRF Annual Invitational Workshop. Montreal, Quebec; March 3, 2005.

5. Langer A, Diaz-Olavarietta C, Berdichevsky K, Villar J. Why is research from developing countries underrepresented in international health literature, and what can be done about it? Bull World Health Organ 2004;82:802-3.

6. Nonaka I, Takeuchi H. The Knowledge Creating Company. How Japanese Companies Create the Dynamics of Innovation. Oxford: Oxford University Press; 1995.

\footnotetext{
${ }^{1}$ World Health Organization, 20 Avenue Appia, 1211 Geneva, Switzerland. Correspondence should be sent to Dr Pablos-Mendez at: pablosa@who.int.

2 National Health Foundation, Bangkok, Thailand.

3 University of the Philippines, Manila, Philippines.

${ }^{4}$ Department of Epidemiology and Community Medicine, University of Ottawa, Canada.
} 